\title{
The reward of a good joke: neural correlates of viewing dynamic displays of stand-up comedy
}

\author{
Robert G. Franklin Jr. • Reginald B. Adams Jr.
}

Published online: 13 July 2011

(C) Psychonomic Society, Inc. 2011

\begin{abstract}
Humor is enjoyable, yet few studies to date have reported that humor engages brain regions involved in reward processing (i.e., the mesolimbic reward system). Even fewer have investigated socially relevant, dynamic displays of real actors telling jokes. Instead, many studies have focused on responses to static cartoons or written jokes in isolation. In the present investigation, we used functional magnetic resonance imaging (fMRI) to examine brain activation in response to video clips of comedians performing stand-up comedy, a more socially relevant task than reading jokes or cartoons in isolation. Participants watched video clips of eight stand-up comedians, half female/half male, that were prerated by a separate group of participants from the same population as eliciting either high or low levels of amusement, thereby allowing us to control for comedian attributes and comedic style. We found that high-funny clips elicited more activation in several brain regions involved with reward responses, including the nucleus accumbens, caudate, and putamen. A regression with participants' own ratings of humor revealed similar activity in reward areas as well as in regions involved in theory of mind. These findings indicate that dynamic social displays of humor do engage reward responses. The rewarding nature of humor may help explain why it is so valued socially.
\end{abstract}

Keywords Amygdala $\cdot$ Emotion · Reward · Prefrontal . Mesolimbic system $\cdot$ Humor $\cdot$ Comedy

R. G. Franklin Jr. R. B. Adams Jr. $(\bowtie)$

Department of Psychology, The Pennsylvania State University, University Park, PA 16802, USA

e-mail: radams@psu.edu
All of us possess a "sense of humor" (admittedly, some more than others), which we use to help us cope with stressful situations, to induce socially lubricating positive emotions, and to defuse tense situations (see, e.g., Dienstbier, 1995; Martin, Kuiper, Olinger, \& Dance, 1993). Humor also plays an important role in social bonding; we like others who are humorous and find them more attractive and appealing (Wanzer, Booth-Butterfield, \& Booth-Butterfield, 1996). From easing tensions, to attracting potential romantic interests, to just having a good time, we use humor in many ways in our social interactions. Humor may serve many purposes, but one of the most difficult questions to answer is why we enjoy it so much. The funny pages in newspapers, specialized genres of television shows, and a multibillion dollar comedy movie industry all attest to our craving for humor. But why is this the case? What is it about humor that makes it so enjoyable? One recent theory (Hurley, Dennett, \& Adams, 2011) has suggested that reward is a central mechanism of humor, motivating a process of debugging inferential errors in our comprehension of the world that is essential for smooth cognitive functioning. Ultimately, this theory hinges on the still thinly explored issue of whether the brain's reward circuitry is essential to experiencing humor.

Several studies have examined the neural regions underlying humor appreciation, showing that the neural "funny bone" is actually a very complex system. Humor requires a coordinated network of responses involved in generating expectations and associations, perceiving incongruities, and revising these expectations, resulting in affective and expressive responses of mirth and laughter. In an early examination of the neural correlates of humor, Goel and Dolan (2001) found a network of brain regions that included the ventromedial prefrontal cortex (vmPFC), temporal regions, and inferior frontal regions. They 
distinguished between temporal regions and inferior frontal regions, which are thought to be primarily involved in humor detection or "getting" a joke, and the vmPFC, which is involved in appreciating a joke. Mobbs, Greicius, Abdel-Azim, Menon, and Reiss (2003) elaborated on this early model using cartoon humor, describing a widespread network involved in the various steps of humor appreciation. This network includes the inferior frontal gyrus, thought to be involved in generating initial expectations; the temporo-occipital junction, involved in detecting incongruity within a humorous episode; and Broca's area and the temporal pole, involved in establishing coherence or resolution of this incongruity. Critically, Mobbs et al. (2003) also found mesolimbic "reward" activation, including in the ventral tegmental area and nucleus accumbens/ventral striatum. In addition, regions likely related to the expression of humor, including the supplementary motor area and dorsal anterior cingulate, were activated.

This complex network is consistent with the incongruityresolution model of humor. In this model, humor activates an initial schema of a situation, followed by a violation of that schema by the introduction of incongruous information, and finally resolution of the incongruity that results in felt mirth see (Suls, 1972). Further supporting this model, Moran, Wig, Adams, Janata, and Kelley (2004), using dynamic displays of humor (i.e., sitcoms), found a temporal relationship between cognitive and affective components of humor as predicted by the incongruity-resolution model, showing activation in the inferior frontal and posterior temporal regions, presumably involved in the initial detection of humor, followed by activation in the insula, presumably related to the actual experience of mirth. Surprisingly, however, they did not find evidence for mesolimbic "reward" activation, as previously reported by Mobbs et al. (2003) when using cartoon humor.

Much of the neuroscience literature indicates that reward and affect encompass related systems (e.g., Ashby, Isen, \& Turken, 1999). However, a separate network of brain regions has been linked to the processing of rewarding stimuli, as compared to those regions often linked to the affective component of humor (Rolls, 1999). Regions related to reward per se include mesolimbic structures such as the nucleus accumbens (NAcc), caudate and putamen, ventral striatum, and ventral tegmental area. Elsewhere, the amygdala and orbitofrontal cortex are also associated with reward. These brain regions comprise a network thought to be involved in assessing the potential rewarding value of stimuli that guide motivational responses involved with seeking reward. The mesolimbic reward system is also critical in reinforcing behavioral responses to appetitive stimuli (Robbins \& Everitt, 1996). Along with processing primary reinforcers like food and sex, the reward system is also involved in processing conditioned rewards such as money (Knutson, Adams, Fong, \& Hommer, 2001), and in processing social reward, such as attractiveness (Aharon et al., 2001; Kampe, Frith, Dolan, \& Frith, 2001; O’Doherty et al., 2003).

The network of brain regions involved in assessing social reward may be highly related to positive affect, since rewarding stimuli usually evoke positive affect as well. Not all positive affect evokes reward responses, however. For instance, Gable and Harmon-Jones (2008) found a dissociation between low-approach positive affect, evoked by cute kittens, and high-approach positive affect, evoked by rewarding stimuli such as cupcakes. The apparent lack of approach motivation in response to some positive stimuli suggests that those stimuli do not evoke typical reward responses, which would be positively reinforcing stimuli that would evoke appetitive/approach responses. Humor may induce positive affect and thus lead to activation in brain regions involved with happiness (such as the insula) without engaging the reward centers. That said, much of what we associate with humor suggests that the involvement of reward centers would be critical.

Only a few studies to date have reported involvement of reward centers in response to experiencing humor. In one study, funny cartoons versus cartoons with their humor content removed elicited more activation in the ventral striatum and nucleus accumbens, as was expected (Mobbs et al., 2003; see also Azim, Mobbs, Jo, Menon, \& Reiss, 2005). Likewise, cartoons rated as funnier elicited activation in reward centers more than did less funny cartoons (Watson, Matthews, \& Allman, 2007). These results suggest that humor does activate reward-related structures. Yet, other studies examining humor have not reported significant activation of the mesolimbic reward system (e.g., Bartolo, Benuzzi, Nocetti, Baraldi, \& Nichelli, 2006; Moran et al., 2004). So far, the only studies that have found consistent mesolimbic reward activation to humor are those focused on cartoons closely matched for content but varying in humor level (either as rated by the participants or prerated by a separate group of participants). Importantly, these studies also had participants focus their attention to their own affective experiences.

Though cartoons are important and popular ways to present humor, they represent only a small percentage of the stimuli we naturally encounter that are considered humorous. Moreover, although static cartoons or newspaper comics may describe social scenes, and may even require theory of mind to process, they nonetheless lack many of the properties of social interaction that are generally part of the humor experience. Indeed, people tend to be the agents of most everyday humor through joke-telling. Perhaps the quintessential example of social humor is the stand-up comedian. Stand-up comedians elicit humor through verbal information, vocal cues, and nonverbal expression such as facial displays and gesture. 
Much of stand-up comedy is based on the theatrical presentation style of the comedian and not simply on the verbal content of the jokes themselves (Stebbens, 1990). Yet, surprisingly, the only study to date to use dynamic video stimuli did not report significant reward activation as previously found in response to cartoons.

Moran et al. (2004) examined time-locked activation to laugh tracks recorded for sitcoms in order to parse cognitive versus affective responses to humor. In this study, the experimenters modeled humor by examining brain activation time-locked to $2 \mathrm{~s}$ prior to a laugh track, for detecting humor, and modeled the time during laughter itself to capture affective humor responses, using the rest of the episode as baseline. Two experiments revealed robust brain activation in regions involved with social processing and positive affect; however, again, they did not find mesolimbic responses. If humor is rewarding, then it is quite surprising that these studies, employing extremely engaging and naturalistic dynamic stimuli, should fail to yield reward-related activation. If anything, we might have expected more robust responses in this situation. That said, given that Moran et al. (2004) examined humor responses within the context of a humorous sitcom, it is likely that reward regions, sensitive to both the experience of and anticipation of reward (Knutson et al., 2001), were active throughout the episode. If so, this activation would have been cancelled out in their contrast to "nonfunny" parts (absent of distinct joke and laughter) of the episode. We suggest that if the comparison were instead made between watching a sitcom versus some other nonhumorous TV show, or between more or less funny episodes - a comparison that more closely approximates previous research examining responses to cartoon humorthen responses in mesolimbic reward centers might have been more apparent. Therefore, we sought to investigate this puzzling discrepancy in mesolimbic response by examining responses to dynamic displays of social humor using a paradigm that more closely approximates that used by the previous studies examining cartoon humor.

We used closely matched clips of stand-up comedians, contrasting between clips prerated for each comedian as high and low in funniness by a sample of undergraduate students from the same population as recruited for the fMRI portion of the study. In this way, we could control specific comedian attributes, such as gender, attractiveness, genre of humor, nonverbal style, and joke execution. We presented these video clips to participants and asked them to rate their own affective responses to each clip, focusing on their affective experience of the humor rather than their evaluation of the quality of the humor. We predicted that regions involved with reward would be more active for high- versus low-funny clips and would also be correlated with participants' own ratings of humor.

\section{Method}

Participants

A group of 20 participants ( 10 female, 10 male) participated in this study. The participants ranged in age from 18 to 35 years $(M=24.6, S D=5.8)$. They signed consent forms approved by the Pennsylvania State University Institutional Review Board and were paid \$20 each for their participation. One participant (female) was dropped due to reporting hearing problems that interfered with hearing the audio tracks of the stimuli, leaving a total of 19 participants.

Stimuli

A total of 64 comedy clips were chosen from online and television sources for eight comedians (four female-Ellen Degeneres, Janeane Garofalo, Kathy Griffin, and Kathleen Madigan - and four male-Dane Cook, Mitch Hedberg, Jerry Seinfeld, and Steven Wright). Each clip consisted of the comedian performing a stand-up comedy routine alone on a stage, in front of an audience. We cropped the length of each clip to be $24 \mathrm{~s}$, and no clip began or ended midsentence. Eight clips by each comedian were pilottested on 22 undergraduates in order to select clips that were rated as high and low in humor. Participants were asked how amusing they personally found the clips and rated the clips on a 7-point scale. From these preratings, four clips were chosen for each comedian, the two rated highest in humor (high humor) and the two rated lowest in humor (low humor). Humor preratings of the clips were highly reliable $(\alpha=.819)$. An items analysis confirmed that the high-humor clips chosen $(M=4.42$ out of 7, $S E=.133)$ were significantly funnier than the low-humor clips $(M=3.26, S E=.112), t(30)=6.675, p<.0001$.

\section{Procedure}

Participants completed two runs of the fMRI task, in which they viewed stand-up comedy clips. Each run consisted of 16 clips of humor alternating between high and low humor. Within each run, two clips of each comedian (one high humor and one low humor) were shown in a pseudorandom order, ensuring that the same comedian did not appear in two adjacent blocks. After each 24-s clip was displayed, participants had $6 \mathrm{~s}$ to rate the clip on how personally amused they were by the clip on a 1-to- 8 scale, anchored by $1=$ not at all amused and $8=$ very amused. Participants entered ratings by using two four-button response pads, one for each hand, and were instructed which button corresponded to which rating (i.e., left pinky $=1$ to right pinky $=8$ ). Following each rating, participants viewed the next clip. Each run lasted $8 \mathrm{~min}$. 
Scanning parameters and analysis

Functional data were collected using a 3 T Siemens TimTrio using echo-planar imaging with two runs of $160 \mathrm{~T} 2 *$ images (TR: $3,000 \mathrm{~ms}$, TE: $25 \mathrm{~ms}, 36$ interleaved slices, transverse orientation, $3.4 \times 3.4 \times 3 \mathrm{~mm}$ voxels, with $3-\mathrm{mm}$ slices, and no gap). We used SPM5 software to process the data (Wellcome Institute, London, U.K.). First, we realigned the data by a six-parameter rigid realignment that generated movement parameters used as regressors for the first-level analysis, to control for artifact activation caused by movement. We then co-registered the mean functional realigned image to a $\mathrm{T} 1$ anatomical image. This anatomical image was then segmented and normalized to the Montreal Neurological Institute (MNI) template. The normalization parameters generated by this normalization were then applied to each functional image. Finally, we smoothed each functional image using an 8-mm full-width-at-half-maximum Gaussian kernel.

Contrasts were initially estimated using a first-level fixed effects model within each participant's data. Stimulus conditions were modeled as delayed boxcar functions convolved with a standard hemodynamic response function. Low-frequency signal components less than $0.007 \mathrm{~Hz}$ were filtered out using a high-pass filter. Contrast images from each participant were then entered into a second-level random-effects model representing summary measures of participant responses. For each participant, we generated first-level contrasts predicting activation for each contrast (i.e., responses to high minus low and low minus high humor). These contrasts were then used for group-level activation.

Activation maps were thresholded at $p<.005$ uncorrected, with a cluster extent of 46 voxels. This threshold corresponded to a $p<.05$ corrected extent in a Monte Carlo simulation using the AlphaSim software included in the
AFNI (Analysis for Functional NeuroImaging) program (Cox, 1996). This simulation was performed using 1,000 iterations and a volume of voxels based on the acquisition mask generated from the group-level random-effects model.

\section{Results}

Manipulation check

A manipulation check confirmed that the preselected highhumor comedy clips $(M=4.62, S D=0.98)$ were rated as significantly more amusing than the low-humor comedy clips $(M=3.81, S D=0.68), t(30)=2.729, p=.011$.

High minus low humor

We contrasted responses to clips prerated as being high versus low in humor. This contrast revealed the predicted activation in reward centers, including the right nucleus accumbens, caudate, and putamen bilaterally (see Fig. 1, left pane). Also active were the left inferior frontal gyrus, the left temporoparietal junction, and the right supplementary motor area (see Table 1). The reverse contrast (responses to low- vs. high-humor comedy clips) revealed no significant activation.

Regression with participants' own humor ratings

We further examined the participants' own rated experiences of humor to these clips. To examine this question, we computed a regression using each participant's ratings of their own experienced amusement after each clip as a trialspecific first-level regressor, while applying inclusionary and exclusionary masks based on the high-minus-low prerated humor contrast. We used an inclusionary mask
Fig. 1 Activation maps for mesolimbic reward in the contrast of high minus low humor using preratings of stimuli (left pane), and for the positive regression of participants' own ratings of humor (right pane). Images are thresholded at $p<.005,46$ voxels

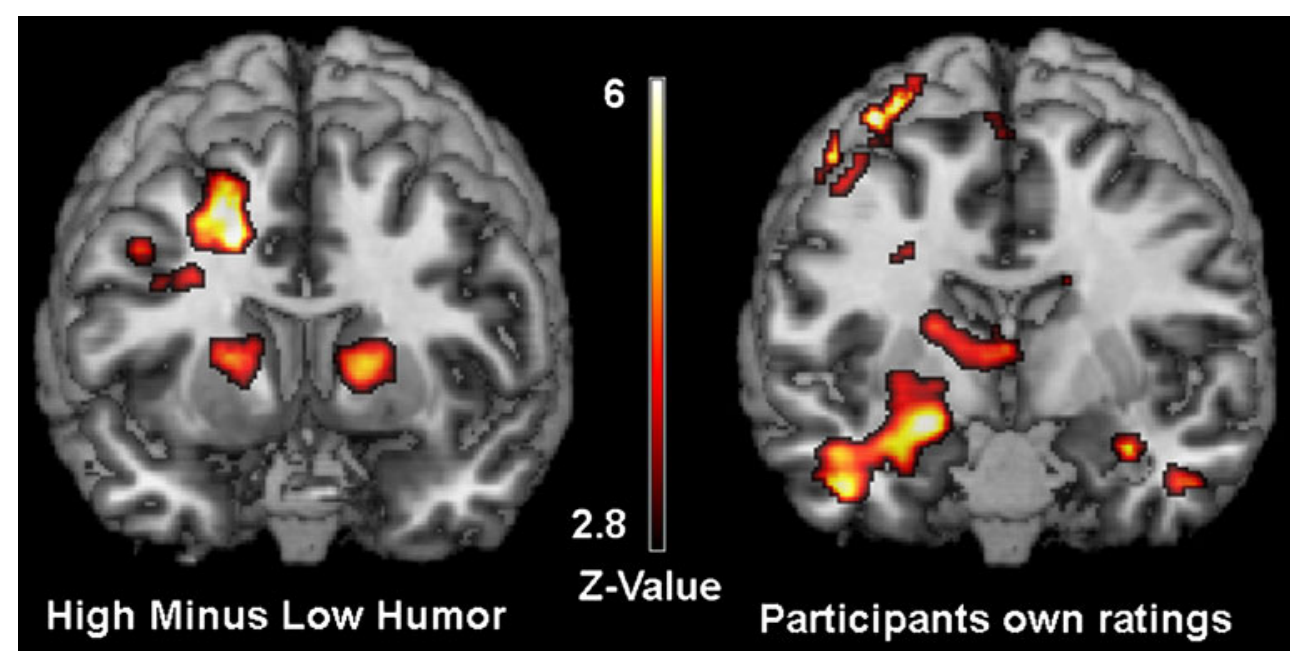


Table 1 Regions active for comedy clips rated as being high versus low in humor

\begin{tabular}{|c|c|c|c|c|c|}
\hline \multirow[b]{2}{*}{ Brain Region } & \multicolumn{3}{|c|}{ MNI Coordinates } & \multirow[b]{2}{*}{$t$ Value } & \multirow[b]{2}{*}{ Extent } \\
\hline & $x$ & $y$ & $z$ & & \\
\hline L. Temporoparietal Junction & -36 & -46 & 22 & 3.88 & 194 \\
\hline R. Caudate & 26 & -10 & 18 & 3.72 & 152 \\
\hline R. Supplementary Motor Area & -18 & 8 & 44 & 5.04 & 1,344 \\
\hline R. Putamen & 16 & 10 & 6 & 3.74 & 157 \\
\hline L. Putamen & -20 & 10 & 10 & 3.66 & 125 \\
\hline L. Inferior Frontal Gyrus & -44 & 20 & 30 & 4.47 & 750 \\
\hline
\end{tabular}

Regions are ordered from posterior to anterior. supplementary motor area, left temporoparietal junction, and right cerebellum (see Table 2, top pane). Next, using the high-minus-low contrast as an exclusionary mask, we found activation in the superior temporal sulcus (STS), inferior temporal lobe, amygdala, and cerebellum (see Table 2, bottom pane). We also found activation in the left primary motor cortex, likely only reflecting activation for motor responses for higher ratings, as these responses were made by the right hand.

Finally, we examined activation in regions for the negative regression of humor ratings, or regions that were more active to ratings of low versus high humor. Given that no regions were active for this contrast when examining clips prerated as low versus high in humor, we did not
Table 2 Regions active for the regressions of brain activation to participants' own ratings of humor in the fMRI task, using activation for comedy pretested as being high versus low in humor as an inclusionary (top) and exclusionary (bottom) mask

*The peak indicated is part of the cluster extent referred to immediately above.

\begin{tabular}{|c|c|c|c|c|c|}
\hline \multirow[b]{2}{*}{ Brain Region } & \multicolumn{3}{|c|}{ MNI Coordinates } & \multirow[b]{2}{*}{$t$ Value } & \multirow[b]{2}{*}{ Extent } \\
\hline & $x$ & $y$ & $z$ & & \\
\hline \multicolumn{6}{|c|}{ Regions Active to the Inclusionary Mask } \\
\hline R. Cerebellum & 24 & -80 & -38 & 3.74 & 174 \\
\hline L. Temporoparietal Junction & -38 & -46 & 22 & 3.83 & 204 \\
\hline L. Precentral Gyrus & -38 & -26 & 48 & 3.56 & 453 \\
\hline L. Putamen & -22 & 0 & 12 & 3.25 & 312 \\
\hline L. Supplementary Motor Area & 0 & 22 & 54 & 3.72 & 108 \\
\hline \multicolumn{6}{|c|}{ Regions Active to the Exclusionary Mask } \\
\hline R. Cerebellum & 26 & -80 & -38 & 5.94 & 266 \\
\hline L. Cerebellum & 20 & -76 & -42 & 4.88 & $*$ \\
\hline R. Striate Cortex & 22 & -78 & 12 & 3.93 & 50 \\
\hline L. Temporoparietal Junction & -52 & -46 & 30 & 4.04 & 131 \\
\hline R. Superior Temporal Sulcus & 68 & -36 & 0 & 3.34 & 127 \\
\hline R. Parahippocampal Cortex & 32 & -32 & 6 & 3.61 & 442 \\
\hline L. Inferior Temporal Gyrus & -46 & -30 & -10 & 5.56 & 441 \\
\hline L. Pons & -14 & -24 & -12 & 4.9 & 4,194 \\
\hline R. Caudate/Putamen & 4 & -6 & 6 & 2.94 & $*$ \\
\hline L. Amygdala & -22 & -4 & -12 & 4.23 & $*$ \\
\hline L. Superior Temporal Sulcus & -50 & 8 & -24 & 5.67 & $*$ \\
\hline L. Primary Motor Cortex & -40 & -22 & 54 & 5.47 & 1,315 \\
\hline R. Amygdala & 34 & -8 & -20 & 4.06 & 583 \\
\hline R. Temporal Pole & 52 & 14 & -14 & 5 & 583 \\
\hline
\end{tabular}


employ the inclusionary and exclusionary mask approach described above, but instead used a whole-brain regression. This contrast yielded activation in the vmPFC, posterior cingulate, and insula (see Table 3 ). We also found activation in the right primary motor cortex, likely reflecting lower ratings made by the left hand.

\section{Discussion}

We investigated neural activation underlying humor specifically as it applies to a naturalistic, dynamic social interaction, to address the puzzling lack of evidence for mesolimbic responses using such dynamic stimuli. The present study examined the neural activation associated with watching stand-up comedians, specifically contrasting high- and low-amusing skits of the same comedians, as selected based on preratings made by a sample of raters from the same student population. Although stand-up comedy is certainly still a performance art, it simulates the joke-telling experience in everyday life, where one person surrounded by others captures the attention of the group and delivers the necessary cognitive structure and elements to produce a mirth response and receive the social capital that comes with it.

To our knowledge, the only other study that has used dynamic stimuli reported no evidence for activation in reward centers (Moran et al., 2004). However, in the present work we found activation of reward centers when contrasting high versus low humor using highly controlled and ecologically valid stimuli. In this case, we used a comparison that more closely matched that employed in studies examining cartoon humor, thereby helping reconcile the apparent discrepancy of the previous findings. This finding demonstrates that dynamic displays of social humor do appear to activate reward centers. We also found activation in several brain regions that have been more consistently implicated in understanding and experiencing humor. These include the inferior frontal cortex and temporoparietal junction (TPJ). Both of these regions have been previously shown to be involved in the detection of humor (Mobbs et al., 2003; Moran et al., 2004). The inferior frontal cortex and TPJ are both considered to be involved with decoding others' mental states (e.g., Frith \& Frith, 1999), which is important for understanding humor. This is especially important in understanding how a joke's punch line often resolves incongruities between the mental state of a subject in a joke and the mental state of the audience of a joke (Bartolo et al., 2006), which may reflect why these regions are active here. The social nature of the present task highlights this possibility. However, it is also possible that these regions could be involved in humor appreciation for other reasons, including the roles of the inferior frontal cortex and TPJ in attentional switching. Both of these regions are active when disengaging from invalid visual cues (e.g., Arrington, Carr, Mayer, \& Rao, 2000), and activity here may reflect the importance in disengaging from mental operations involved with incongruities that are linked to humor.

We also examined a regression using the fMRI participants' own ratings of humor. Although this analysis lacks the control of our direct comparison, it also more precisely reflects participants' own subjective experiences while in the magnet. This analysis revealed many of the same regions active as found in the direct contrast of prerated high versus low humor, including the mesolimbic reward regions including the left putamen, as well as regions involved in mental state decoding such as the TPJ. Additionally, we found activation in other regions that were not active for the clips prerated as high versus low in humor. These regions also included reward regions such as bilateral caudate and putamen, as well as the amygdala. Additional regions involved in mental state inference were active for this contrast as well, including the temporal poles and STS. Both of these regions are known to be involved with deciphering what others are thinking, with the STS specifically involved with decoding information important to the intent of others, such as biological motion, facial emotion, and eye gaze, while the temporal poles are implicated in recalling socially relevant memory, such as social scripts (Allison, Puce, \& McCarthy, 2000; Gallagher \& Frith, 2003). The parahippocampal cortex was also active, replicating previous similar findings (e.g., Moran et al., 2004; Watson et al., 2007), which might be due to memory of social scripts that are then violated as part of the expectancy violation model of humor.
Table 3 Regions active for the negative regression (low minus high) for participants' own ratings of comedy

\begin{tabular}{|c|c|c|c|c|c|}
\hline \multirow[b]{2}{*}{ Brain Region } & \multicolumn{3}{|c|}{ MNI Coordinates } & \multirow[b]{2}{*}{$t$ Value } & \multirow[b]{2}{*}{ Extent } \\
\hline & $x$ & $y$ & $z$ & & \\
\hline R. Precentral Gyrus & 42 & -22 & 56 & 8.59 & 1,605 \\
\hline R. Insula & 46 & -20 & 20 & 5.62 & 341 \\
\hline R. Cingulate Gyrus & 10 & -18 & 46 & 3.68 & 89 \\
\hline Ventromedial Prefrontal Cortex & 0 & 18 & -12 & 6.01 & 2,661 \\
\hline
\end{tabular}


We also found activation reflecting a negative association between humor experience ratings and the insula, posterior cingulate, and vmPFC. This seems surprising, given that the vmPFC is known to be involved in decoding humor (e.g., Goel \& Dolan, 2001) via understanding others' mental states (e.g., Mitchell, Banaji, \& Macrae, 2005). It is possible that activation in the vmPFC may reflect greater attempts to understand the humor in the clips rated as less amusing. Additionally, the posterior cingulate, insula, and vmPFC are also regions implicated as part of the default network of the brain, a network involved in stimulus-independent thought and frequently active in rest periods of fMRI experiments (Buckner, Andrews-Hanna, \& Schacter, 2008). Indeed, activity in each of these three regions has been positively correlated with the amount to which participants' minds wander during an fMRI experiment (Mason et al., 2007). Thus, activation in these regions for this contrast may simply reflect participants' reduced engagement in the task while watching low-humor clips.

Humor studies have found inconsistent results for eliciting reward activation. One possible explanation is that studies finding reward activation, including the present study, have emphasized the affective response or the feeling involved with humor. Other studies, focusing more on the quality of humor, have failed to find reward activity, presumably because of the focus on more cognitive aspects of humor (e.g., Bartolo et al., 2006). However, this difference does not explain all studies that have not reported reward-related activation. For instance, Moran et al. (2004) had participants passively view a sitcom and examined humor responses locked to a laugh track. Participants' humor responses were thus measured in the context of an ongoing humorous episode, where humor was anticipated at any moment in the episode. This consistent anticipated reward might have led to mesolimbic activation in other portions of the episode, which thus would have been subtracted out of any direct comparison. Therefore, the high level of control offered between high and low humor, as well as the sensitivity offered by examining participants' own ratings, may have been key to our finding mesolimbic activation in the present study. What is clear in the present study is that funnier dynamic joke-telling yielded greater activation in the reward network, as expected, and as previously found when examining cartoon humor using a similarly controlled paradigm. These findings, therefore, offer further evidence consistent with current theories that critically hinge on the presumption that reward responses are fundamental to the humor process (e.g., Hurley et al., 2011).

Interestingly, this work seems to indicate that the social humor we find funniest also involves activation of brain regions required to process social intent. Several humor studies using static cartoons have found activation in brain regions involved with decoding what others are thinking, such as the temporal poles, TPJ, and STS (e.g., Azim et al., 2005; Goel \& Dolan, 2001; Mobbs, Hagan, Azim, Menon, $\&$ Reiss, 2005). These areas are more active for cartoons that involve taking others' perspectives into account (e.g., Samson, Zysset, \& Huber, 2008), a finding that highlights their importance in incorporating knowledge of others' mental states in humor. The study here, using dynamic displays of humor, revealed that brain regions involved with decoding the social intent of others are related to greater humor experience, perhaps reflecting the processing of incongruity between social actions and expectations.

This study builds upon Moran et al.'s (2004) findings by indicating that more naturalistic humor is indeed perceived and processed as a rewarding stimulus. We enjoy humor from others, and humor plays a role in how we judge them (Wanzer et al., 1996). This may be because we find humor rewarding and associate that reward with those who produce it. This may help address why humor is generally regarded as an important social skill. A parallel may be drawn with perceived attractiveness. Physical attractiveness in others activates many of the same reward brain regions as humor (Aharon et al., 2001; O'Doherty et al., 2003), and attractiveness is likewise associated with positive halo effects and increased liking (e.g., Dion, Berscheid, \& Walster, 1972). Likewise, reward activation associated with humor may contribute to why we like those who are funny, though this remains an empirical question that warrants future research.

In sum, this article reveals that ecologically relevant, dynamic displays of humor do elicit reward-related activation in the brain. This extends prior research of this kind by showing that more socially relevant forms of humor induce brain activation in much the same way as cartoons do when subjected to similar experimental controls, and it may offer insight as to why humor is so critical a social skill.

Author note This research was supported by a Social Science Research Institute grant, Penn State University, to R.B.A. Jr. We acknowledge the help of Joseph Beeney, Cara Henry, David Pennell, and Kathleen VanHorn in data collection and analysis, and thank Matthew M. Hurley for reading and commenting on an earlier draft of the manuscript.

\section{References}

Aharon, I., Etcoff, N., Ariely, D., Chabris, C. F., O'Connor, E., \& Breiter, H. C. (2001). Beautiful faces have variable reward value: fMRI and behavioral evidence. Neuron, 32, 537-551.

Allison, T., Puce, A., \& McCarthy, G. (2000). Social perception from visual cues: Role of the STS region. Trends in Cognitive Sciences, 4, 267-278. doi:10.1016/S1364-6613(00)01501-1. 
Arrington, C. M., Carr, T. H., Mayer, A. R., \& Rao, S. M. (2000). Neural mechanisms of visual attention: Object-based selection of a region in space. Journal of Cognitive Neuroscience, 12(Suppl. 2), 106-117. doi:10.1162/089892900563975.

Ashby, F. G., Isen, A. M., \& Turken, A. U. (1999). A neuropsychological theory of positive affect and its influence on cognition. Psychological Review, 106, 529-550.

Azim, E., Mobbs, D., Jo, B., Menon, V., \& Reiss, A. L. (2005). Sex differences in brain activation elicited by humor. Proceedings of the National Academy of Sciences, 102, 16496-16501. doi:10.1073/pnas.0408456102.

Bartolo, A., Benuzzi, F., Nocetti, L., Baraldi, P., \& Nichelli, P. (2006). Humor comprehension and appreciation: an FMRI study. Journal of Cognitive Neuroscience, 18, 1789-1798.

Buckner, R., Andrews-Hanna, J. R., \& Schacter, D. L. (2008). The brain's default network: Anatomy, function, and relevance to disease. Annals of the New York Academy of Sciences, 1124, 138.

Cox, R. W. (1996). AFNI: software for analysis and visualization of functional magnetic resonance neuroimages. Computers and Biomedical Research, 29, 162-173.

Dienstbier, R. A. (1995). The impact of humor on energy, tension, task choices, and attributions: Exploring hypotheses from toughness theory. Motivation and Emotion, 19, 255-268.

Dion, K. K., Berscheid, E., \& Walster, E. (1972). What is good is beautiful. Journal of Personality and Social Psychology, 24, 285-290.

Frith, C. D., \& Frith, U. (1999). Interacting minds-A biological basis. Science, 286, 1692-1695. doi:10.1126/science.286.5445.1692.

Gable, P. A., \& Harmon-Jones, E. (2008). Approach-motivated positive affect reduces breadth of attention. Psychological Science, 19, 476-482. doi:10.1111/j.1467-9280.2008.02112.x.

Gallagher, H. L., \& Frith, C. D. (2003). Functional imaging of "theory of mind. Trends in Cognitive Sciences, 7, 77-83. doi:10.1016/ S1364-6613(02)00025-6.

Goel, V., \& Dolan, R. J. (2001). The functional anatomy of humor: Segregating cognitive and affective components. Nature Neuroscience, 4, 237-238. doi:10.1038/85076.

Hurley, M. M., Dennett, D. C., \& Adams, R. B., Jr. (2011). Inside jokes: Using humor to reverse-engineer the mind. Cambridge, MA: MIT Press.

Kampe, K. K. W., Frith, C. D., Dolan, R. J., \& Frith, U. (2001). Reward value of attractiveness and gaze. Nature, 413, 589-590. doi: $10.1038 / 35098149$.
Knutson, B., Adams, C. M., Fong, G. W., \& Hommer, D. (2001). Anticipation of increasing monetary reward selectively recruits nucleus accumbens. Journal of Neuroscience, 21, RC159.

Martin, R. A., Kuiper, N. A., Olinger, L. J., \& Dance, K. A. (1993). Humor, coping with stress, self-concept, and psychological well-being. Humor, 6, 89-104.

Mitchell, J. P., Banaji, M. R., \& Macrae, C. N. (2005). The link between social cognition and self-referential thought in the medial prefrontal cortex. Journal of Cognitive Neuroscience, 17, 1306-1315.

Mobbs, D., Greicius, M. D., Abdel-Azim, E., Menon, V., \& Reiss, A. L. (2003). Humor modulates the mesolimbic reward centers. Neuron, 40, 1041-1048.

Mobbs, D., Hagan, C. C., Azim, E., Menon, V., \& Reiss, A. L. (2005). Personality predicts activity in reward and emotional regions associated with humor. Proceedings of the National Academy of Sciences, 102, 16502-16506.

Moran, J. M., Wig, G. S., Adams, R. B., Jr., Janata, P., \& Kelley, W. M. (2004). Neural correlates of humor detection and appreciation. NeuroImage, 21, 1055-1060. doi:10.1016/j.neuroimage.2003.10.017.

O’Doherty, J., Winston, J., Critchley, H., Perrett, D., Burt, D. M., \& Dolan, R. J. (2003). Beauty in a smile: the role of medial orbitofrontal cortex in facial attractiveness. Neuropsychologia, 41, 147-155.

Robbins, T. W., \& Everitt, B. J. (1996). Neurobehavioural mechanisms of reward and motivation. Current Opinion in Neurobiology, 6, 228-236. doi:10.1016/S0959-4388(96)80077-8.

Rolls, E. T. (1999). The brain and emotion. Oxford: Oxford University Press

Samson, A. C., Zysset, S., \& Huber, O. (2008). Cognitive humor processing: Different logical mechanisms in nonverbal cartoonsAn fMRI study. Social Neuroscience, 3, 125-140. doi:10.1080/ 17470910701745858.

Stebbins, R. A. (1990). The laugh-makers: Stand-up comedy as art, business, and life-style. Montreal: McGill-Queen's University Press.

Suls, J. M. (1972). A two-stage model for the appreciation of jokes and cartoons. In J. H. Goldstein \& P. E. McGhee (Eds.), Psychology of humor: Theoretical perspectives and empirical issues (pp. 81-100). New York: Academic Press.

Wanzer, M. B., Booth-Butterfield, M., \& Booth-Butterfield, S. (1996). Are funny people popular? An examination of humor orientation, loneliness, and social attraction. Communication Quarterly, 44, $42-52$.

Watson, K. K., Matthews, B. J., \& Allman, J. M. (2007). Brain activation during sight gags and language-dependent humor. Cerebral Cortex, 17, 314-324. 